\title{
Role of break-up processes in fusion enhancement of drip-line nuclei at energies below the Coulomb barrier
}

\author{
K. Hagino, ${ }^{1}$ A. Vitturi, ${ }^{2}$ C.H. Dasso,${ }^{3}$ and S.M. Lenzi ${ }^{2}$ \\ ${ }^{1}$ Institute for Nuclear Theory, Department of Physics, University of Washington, Seattle, WA98195 \\ ${ }^{2}$ Dipartimento di Fisica, Università di Padova and INFN, Padova, Italy \\ ${ }^{3}$ Departamento de Física Atómica, Molecular y Nuclear, Universidad de Sevilla, Spain
}

(July 26, 2021)

\begin{abstract}
We carry out realistic coupled-channels calculations for ${ }^{11} \mathrm{Be}+{ }^{208} \mathrm{~Pb}$ reaction in order to discuss the effects of break-up of the projectile nucleus on sub-barrier fusion. We discretize in energy the particle continuum states, which are associated with the break-up process, and construct the coupling form factors to these states on a microscopic basis. The incoming boundary condition is employed in solving coupled-channels equations, which enables us to define the flux for complete fusion inside the Coulomb barrier. It is shown that complete fusion cross sections are significantly enhanced due to the couplings to the continuum states compared with the no coupling case at energies below the Coulomb barrier, while they are hindered at above barrier energies.
\end{abstract}

Quantum tunneling in systems with many degrees of freedom [1] has attracted much interest in recent years in many fields of physics and chemistry [2]. In nuclear physics, heavy-ion fusion reactions at energies near and below the Coulomb barrier are typical examples for this phenomenon. In order for fusion processes to take place, the Coulomb barrier created by the cancellation between the repulsive Coulomb force and the attractive nuclear interaction has to be overcome. It has by now been well established that the coupling of the relative motion of the colliding nuclei to nuclear intrinsic excitations as well as to transfer reaction channels cause large enhancements of the fusion cross section at subbarrier energies over the predictions of a simple barrier penetration model [3].

The effect of break-up processes on fusion, on the other hand, has not yet been understood very well, and many questions have been raised during the last few years both from the experimental [4 8] and theoretical [9] 12] points of view. The issue has become especially relevant in recent years due to the increasing availability of radioactive beams. These often involve weakly-bound systems close to the drip lines for which the possibility of projectile dissociation prior to or at the point of contact cannot be ignored. Different theoretical approaches to the problem have led to controversial results, not only quantitatively but also qualitatively. The probability for fusion at energies below the barrier has been in fact predicted to be either reduced [9, 10] or enhanced [11, 12] by the coupling to the continuum states.

These investigations, however, were not satisfactory in view of the rather simplified assumptions used in the treatment of both the structure and reaction aspects of the problem. In refs. 99.10] the coupling to the break-up channels was incorporated in terms of a "survival factor", a procedure that underestimates the effects of the coupling in the classically forbidden region, i.e. the dynamical modulation of the effective potential which is most relevant at energies below the barrier. Ref. [11] took this effect into account but the entire continuum space was mocked up by a single discrete configuration and an arbitrary function was introduced to parametrize the radial dependence of the couplings to such state.

In this letter we address the problem without resorting to these approximations. Realistic form factors to the continuum states are constructed by folding the external nuclear and Coulomb fields with the proper singleparticle transition densities, obtained by promoting the last weakly-bound nucleon to the continuum states [13]. The reaction mechanism is described within a full quantal coupled-channel description [14]. The flux for complete fusion is separated inside the Coulomb barrier from that for incomplete fusion using the incoming boundary conditions [15]. In order to isolate the genuine effect of the break-up process, we include only the continuum states in the coupling scheme, neglecting continuum-continuum coupling as well as other inelastic channels such as bound excited states in either reaction partner. For the same reason, we do not take into account static modifications on the ion-ion potential which may arise from the halo properties of the projectile 16 .

As an example for our study we choose the fusion reaction ${ }^{11} \mathrm{Be}+{ }^{208} \mathrm{~Pb}$, where the projectile is generally regarded as a good example of a system with a single neutron "halo". In a pure single-particle picture, the last neutron in ${ }^{11} \mathrm{Be}$ occupies the $2 s_{1 / 2}$ state, bound by about $500 \mathrm{keV}$. The strong concentration of strength at the continuum threshold evidenced in break-up reactions 17] has been mainly ascribed to the promotion of this last bound neutron to the continuum of $p_{3 / 2}$ and $p_{1 / 2}$ states at energy $E_{c}$ via the dipole field [17, 18]. Since the presence of the first excited $1 p_{1 / 2}$ state (still bound by about $180 \mathrm{keV}$ ) may perturb the transition to the corresponding $p_{1 / 2}$ states in the continuum [19], we prefer here to consider only the contribution to the $p_{3 / 2}$ states. The initial $2 s_{1 / 2}$ bound state and the continuum $p_{3 / 2}$ states are generated by Woods-Saxon single-particle potentials 
whose depths have been adjusted to reproduce the correct binding energies for the $1 p_{3 / 2}$ and $2 s_{1 / 2}$ bound states. In particular, one needs for the latter case a potential which is much deeper than the "standard" one. The form factor $F\left(r ; E_{c}\right)$ as a function of the internuclear separation $r$ and of the energy $E_{c}$ in the continuum is then obtained by folding the corresponding transition density with the external field generated by the target. In addition to the Coulomb field, a Woods-Saxon nucleon-nucleus potential is used, with parameters of $R=r_{\circ} A_{T}^{1 / 3}, r_{\circ}=1.27 \mathrm{fm}$, $a=0.67 \mathrm{fm}, V=(-51+33(N-Z) / A) \mathrm{MeV}$, and $V_{l s}=-0.44 \mathrm{~V}$.

The dipole form factor $F\left(r ; E_{c}\right)$ thus constructed at several values of $r$ and $E_{c}$ are shown in Fig. 1. In Fig. 1(a), we display the form factor as a function of $r$ for a fixed value of the energy in the continuum $\left(E_{c}\right.$ being 0.9 $\mathrm{MeV})$. Note the long tail of the nuclear contribution as a consequence of the large radial extension of the weaklybound wave function, resulting in the predominance of the nuclear form factor up to the unusual distance of about $25 \mathrm{fm}$. The same reason gives rise to a deviation of the Coulomb part from the asymptotic behavior proportional to $r^{-2}$. Note also the constructive interference of the nuclear and Coulomb parts, due to the negative E1 effective charge of the neutron excitation. In figs. 1(b) and 1(c), we show, instead, the energy dependence of the form factors for a fixed value of $r$. At large values of $r$ the curves are peaked at very low energies, reflecting the corresponding behavior of the $B(E 1)$. At distances around the barrier (which are most relevant to the fusion process) the peaks of the distributions move to higher energies, especially for the nuclear part.

In order to perform the coupled-channel calculation the distribution of continuum states is discretized in bins of energy, associating to each bin the form factor corresponding to its central energy. We have considered the continuum distribution up to $2 \mathrm{MeV}$, with a step of 200 $\mathrm{keV}$. In this way, the calculations are performed with 10 effective excited channels. The ion-ion potential is assumed to have a Woods-Saxon form with parameters $V_{\circ}=-152.5 \mathrm{MeV}, r_{\circ}=1.1 \mathrm{fm}$ and $a=0.63 \mathrm{fm}$, a set that leads to the same barrier height as the Akyüz-Winther potential. At the distance inside the Coulomb barrier where the incoming boundary conditions are imposed, the flux for the entrance channel and the excited breakup channels are evaluated separately. Cross sections for complete fusion, leading to ${ }^{219} \mathrm{Rn}$, are then defined using only the flux for the entrance channel, while those for incomplete fusion which leads to ${ }^{218} \mathrm{Rn}$ are defined in terms of the flux for the break-up channels [15.

Figure 2(a) shows the results of our calculations. The solid line represents the complete fusion cross section, while the dashed line denotes the sum of the complete and incomplete fusion cross sections. Also shown for comparison, by the thin solid line, is the cross section in the absence of the couplings to the continuum states. One can see that these enhance the fusion cross sections at energies below the barrier over the predictions of a one- dimensional barrier penetration model. Note that this is the case not only for the total (complete plus incomplete) fusion probability, but also for the complete fusion in the entrance channel. This finding is qualitatively the same as that of Ref. 12 which used a three-body model to reach to this conclusion, and supports the results of the original calculation performed in Ref. [11]. As it has been emphasized there, accounting properly for the dynamical effects of the coupling in the classically forbidden region is essential to arrive to this conclusion.

The situation is completely reversed at energies above the barrier, where fusion in the break-up channel becomes more important and dominates at the expense of the complete fusion. As a consequence, the cross sections for complete fusion are hindered when compared with the no-coupling case. It is interesting to check if this hindrance is caused mainly by the Coulomb interaction. Figure 2(b) shows the effect of the individual nuclear and Coulomb excitations separately. It is apparent from this figure that the nuclear coupling plays a more important role than the Coulomb one in fusion reactions at energies below the Coulomb barrier. This can be understood by considering that the nuclear process is essentially dominated by the values of the potentials and couplings around the Coulomb barrier. At energies above the barrier, on the other hand, both the Coulomb and the nuclear break-up processes play an important role in suppressing the fusion cross sections. This is a characteristic feature of loosely bound systems, where the nuclear form factor extends outside the Coulomb barrier. For fusion of stable nuclei, the Coulomb break-up would become more important in complete fusion at energies above the barrier.

In summary, we have performed exact coupledchannels calculations for weakly-bound systems using realistic coupling form factors to discuss effects of breakup on subbarrier fusion reactions. As an example, we have considered the fusion of ${ }^{11} \mathrm{Be}$ with a ${ }^{208} \mathrm{~Pb}$ target, taking into account the dipole transition of the weaklybound $2 s_{1 / 2}$ neutron to the $p_{3 / 2}$ continuum, which gives the dominant contribution to the low-energy $B(E 1)$ response. Couplings to bound excited states both in the projectile and in the target nuclei as well as the static change of the ion-ion potential were left aside in order to investigate genuine effects of the break-up processes on fusion reactions. We find that the coupling to break-up channels enhances cross sections for the complete fusion at energies below the Coulomb barrier, while it reduces them at energies above.

Very recently, a complete fusion excitation function was measured for the ${ }^{9} \mathrm{Be}+{ }^{208} \mathrm{~Pb}$ reaction at nearbarrier energies by Dasgupta et al. [6]. They showed that cross sections for complete fusion are considerably smaller at above-barrier energies compared with a theoretical calculation that reproduces the total fusion cross section, in general agreement with our results. We note, however, that it is not at all easy to identify reliable reference measurements to compare with at energies below 
the barrier. This feature may make it quite difficult to settle the issue on a purely experimental basis.

The authors are grateful to the ECT* in Trento, the INT at the University of Washington (A.V.), and the INFN Padova (K.H.) for their hospitality and for partial support for this project.

[1] A.O. Caldeira and A.J. Leggett, Phys. Rev. Lett. 46, 211 (1981).

[2] Proceedings of the Fourth International Symposium on Foundations of Quantum Mechanics, edited by M. Tsukada et al., Japanese Journal of Applied Physics Series Vol. 9 (Publication Office of Japanese Journal of Applied Physics, Tokyo, 1993).

[3] C.H. Dasso, S. Landowne and A. Winther, Nucl. Phys. A405, 381 (1983);

M. Dasgupta, D.J. Hinde, N. Rowley, and A.M. Stefanini, Annu. Rev. Nucl. Part. Sci. 48, 401 (1998);

A.B. Balantekin and N. Takigawa, Rev. Mod. Phys. 70, 77 (1998).

[4] K.E. Rehm et al., Phys. Rev. Lett. 81, 3341 (1998).

[5] J. Takahashi et al., Phys. Rev. Lett. 78, 30 (1997).

[6] M. Dasgupta et al., Phys. Rev. Lett. 82, 1395 (1999).

[7] C. Signorini et al., Eur. Phys. J. A 2, 227 (1998).

[8] J.J. Kolata et al., Phys. Rev. Lett.81, 4580(1998); P.A. De Younget al., Phys. Rev. C58, 3442(1998).

[9] N. Takigawa, M. Kuratani and H. Sagawa, Phys. Rev. C47, R2470 (1993).

[10] M.S. Hussein, M.P. Pato, L.F. Canto and R. Donangelo, Phys. Rev. C46, 377 (1992).

[11] C.H. Dasso and A. Vitturi, Phys. Rev. C50, R12 (1994).
[12] K. Yabana, Prog. Theo. Phys. 97, 437 (1997).

[13] C.H. Dasso, S.M. Lenzi and A. Vitturi, Nucl. Phys. A639, 635 (1998).

[14] K. Hagino, N. Rowley, and A.T. Kruppa, Comp. Phys. Comm., in press.

[15] K. Hagino and N. Takigawa, Phys. Rev. C58, 2872 (1998).

[16] N. Takigawa and H. Sagawa, Phys. Lett. B265, 23 (1991).

[17] T. Nakamura et al., Phys. Lett. B331, 296 (1994); T. Nakamura et al., Phys. Lett. B394, 11 (1997).

[18] H. Esbensen, G.F. Bertsch and C.A. Bertulani, Nucl Phys. A581, 107 (1995).

[19] C.H. Dasso, S.M. Lenzi and A. Vitturi, Phys. Rev.C59, 539 (1999).

FIG. 1. Coupling form factor $F\left(r ; E_{c}\right)$ associated with the dipole transition in ${ }^{11} \mathrm{Be}$ from the neutron bound state $2 s_{1 / 2}$ $\left(E_{b}=-500 \mathrm{keV}\right)$ to the continuum state $p_{3 / 2}$ with energy $E_{c}$. In (a) the Coulomb (dot-dashed line), nuclear (dashed) and total (solid) form factors are shown as a function of $r$ at the continuum energy $E_{c}=0.9 \mathrm{MeV}$. In (b) and (c) the form factors are shown as a function of the energy $E_{c}$ in the continuum for $r=r_{\text {barrier }}=11.6 \mathrm{fm}$ and $r=30 \mathrm{fm}$, respectively.

FIG. 2. (a) Fusion cross section for the reaction ${ }^{11} \mathrm{Be}+$ ${ }^{208} \mathrm{~Pb}$ as a function of the bombarding energy in the center of mass frame. The thin solid curve shows the results of the one-dimensional barrier penetration as a reference. The solid and the dashed lines are solutions of the coupled-channels equations for the complete fusion and the complete plus incomplete fusion, respectively. (b) Complete fusion cross sections obtained by including only the nuclear (dashed) and the Coulomb (dot-dashed) couplings, in comparison with the case where both the couplings are considered (solid). 


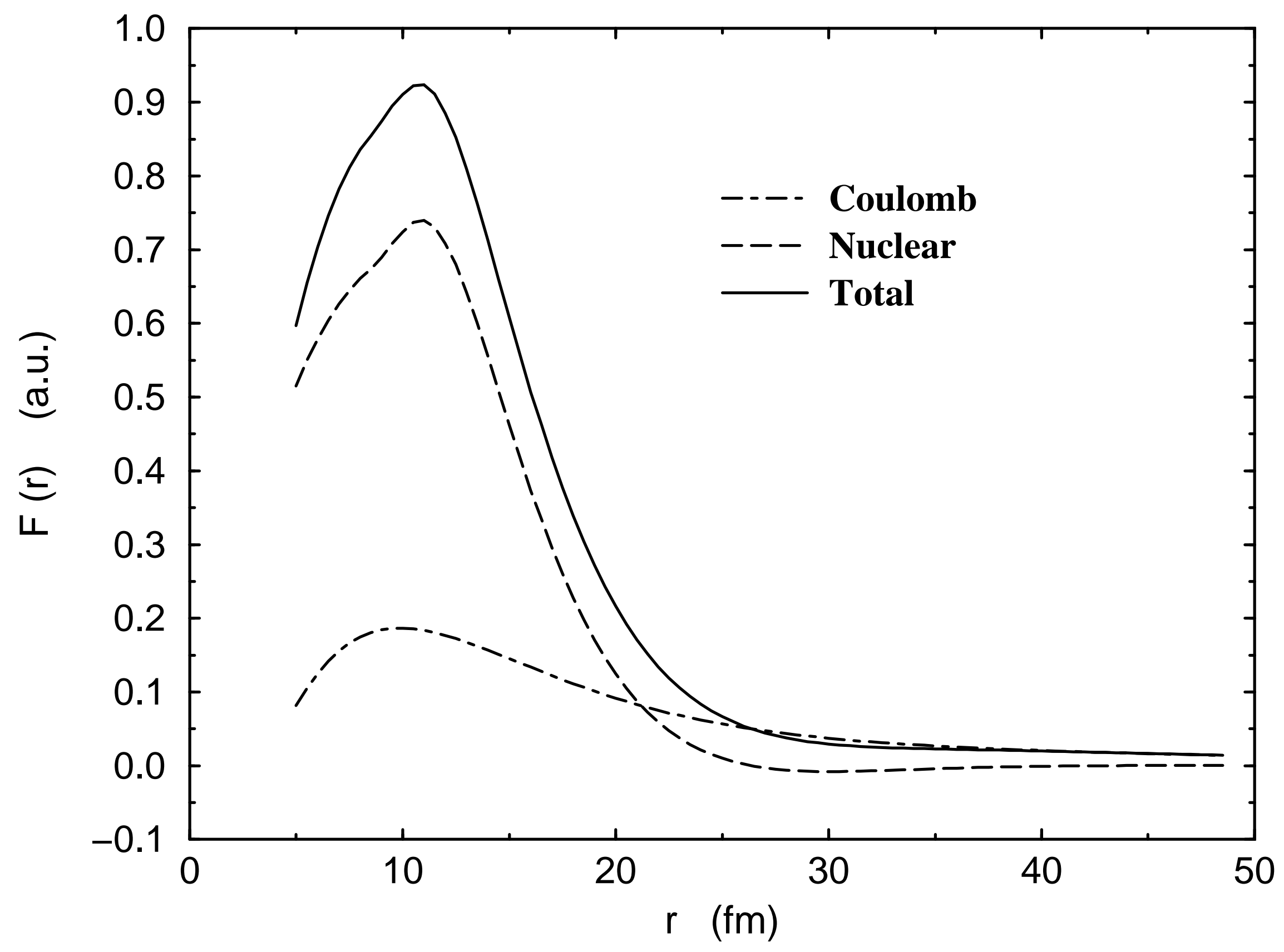




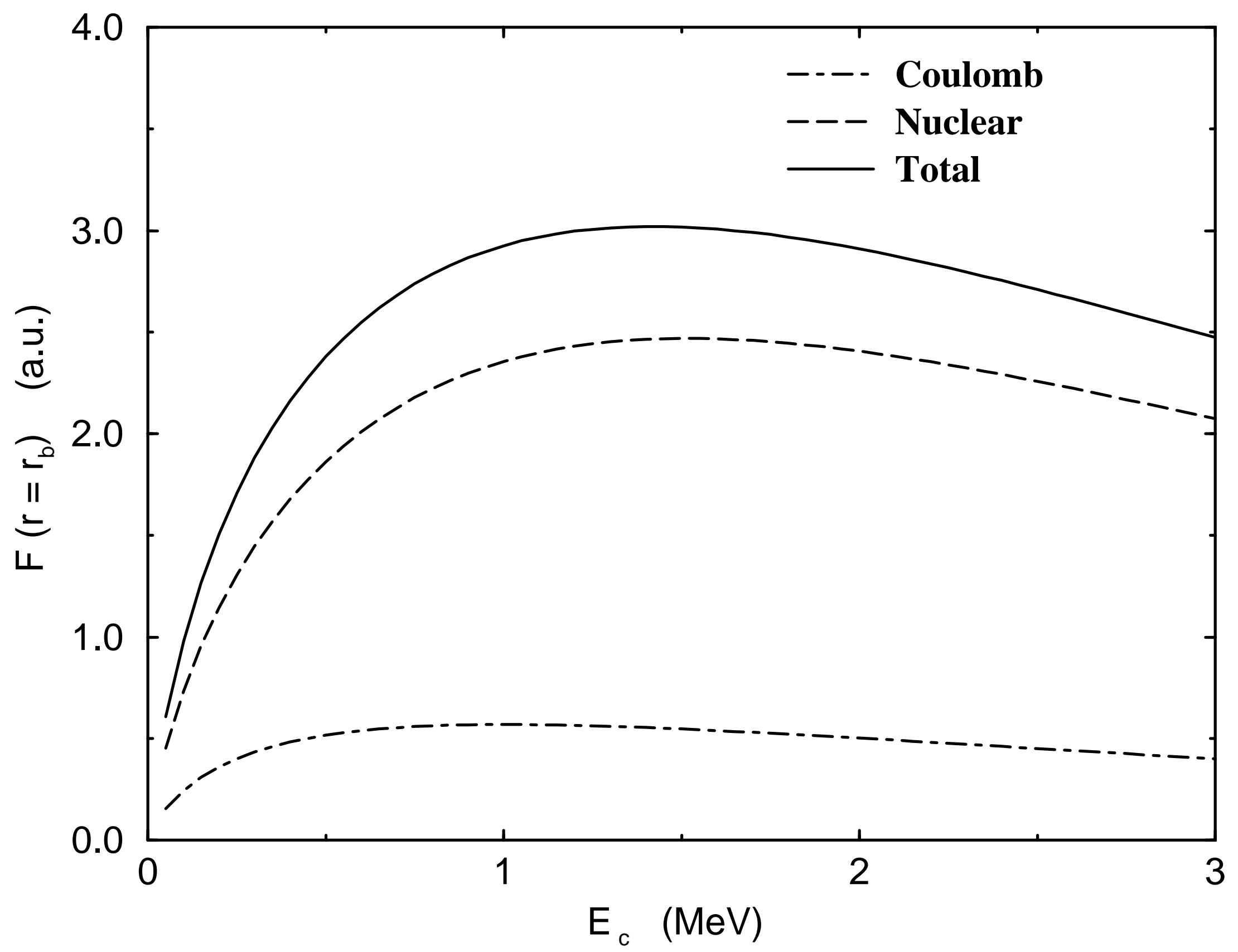




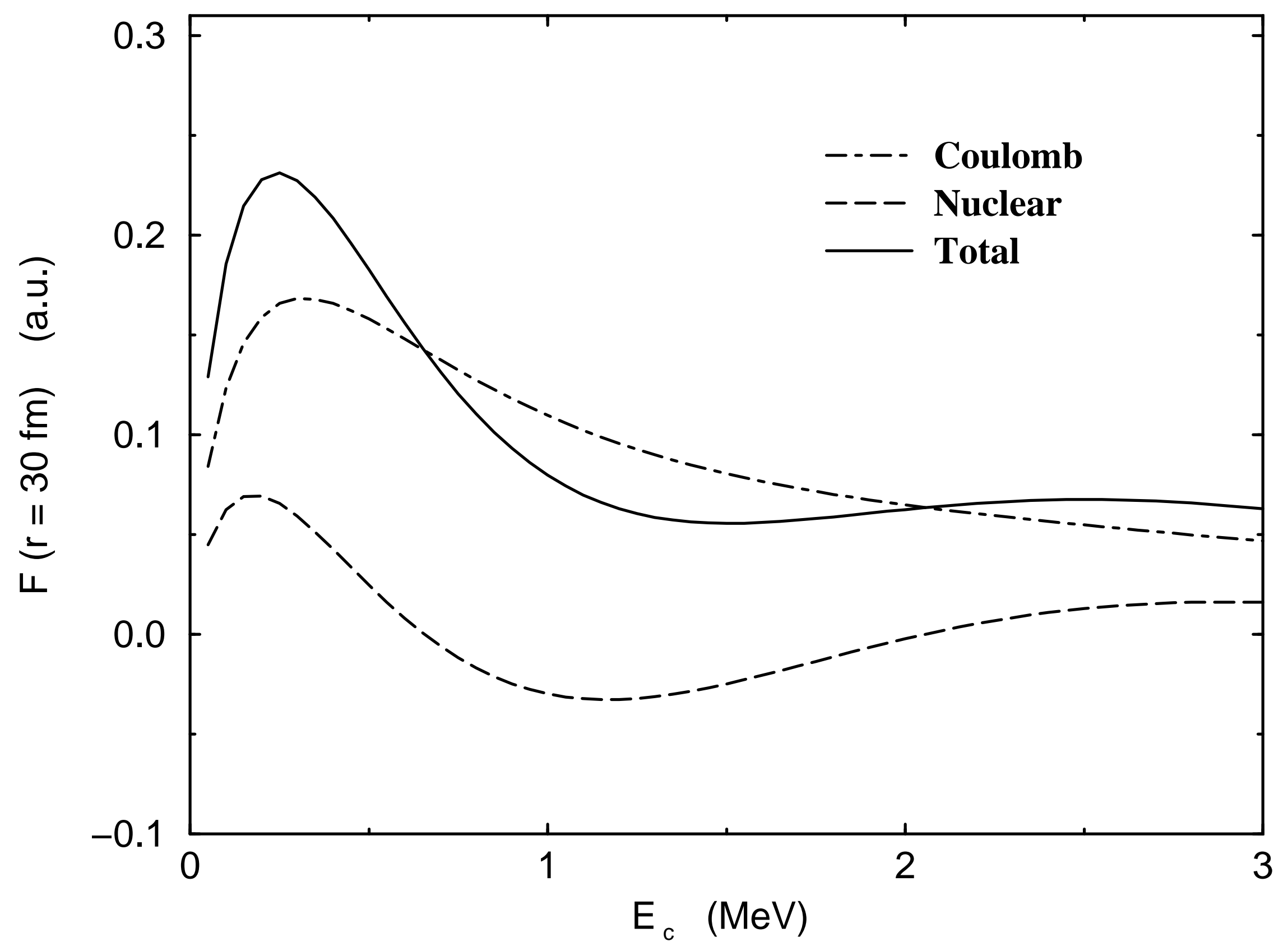




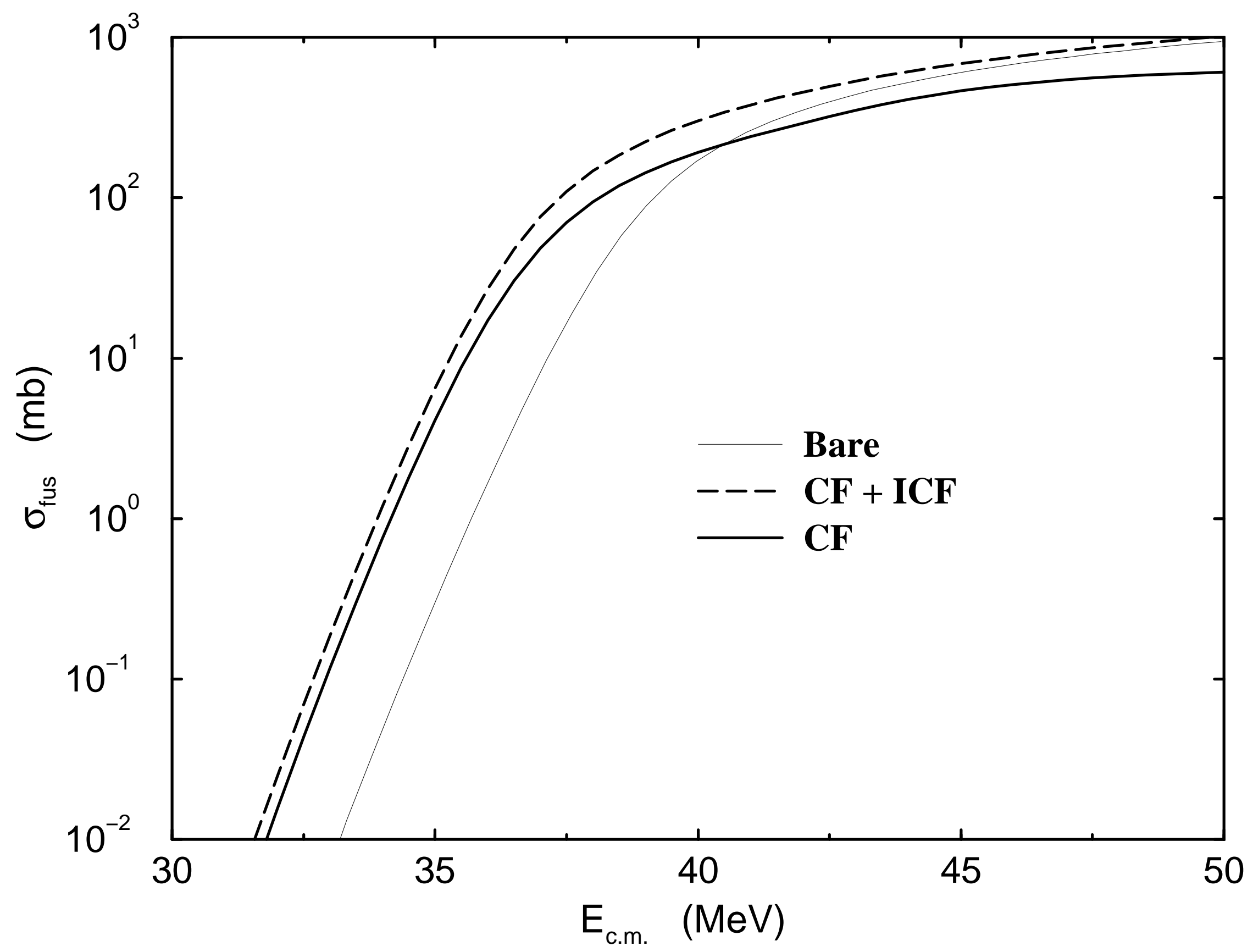




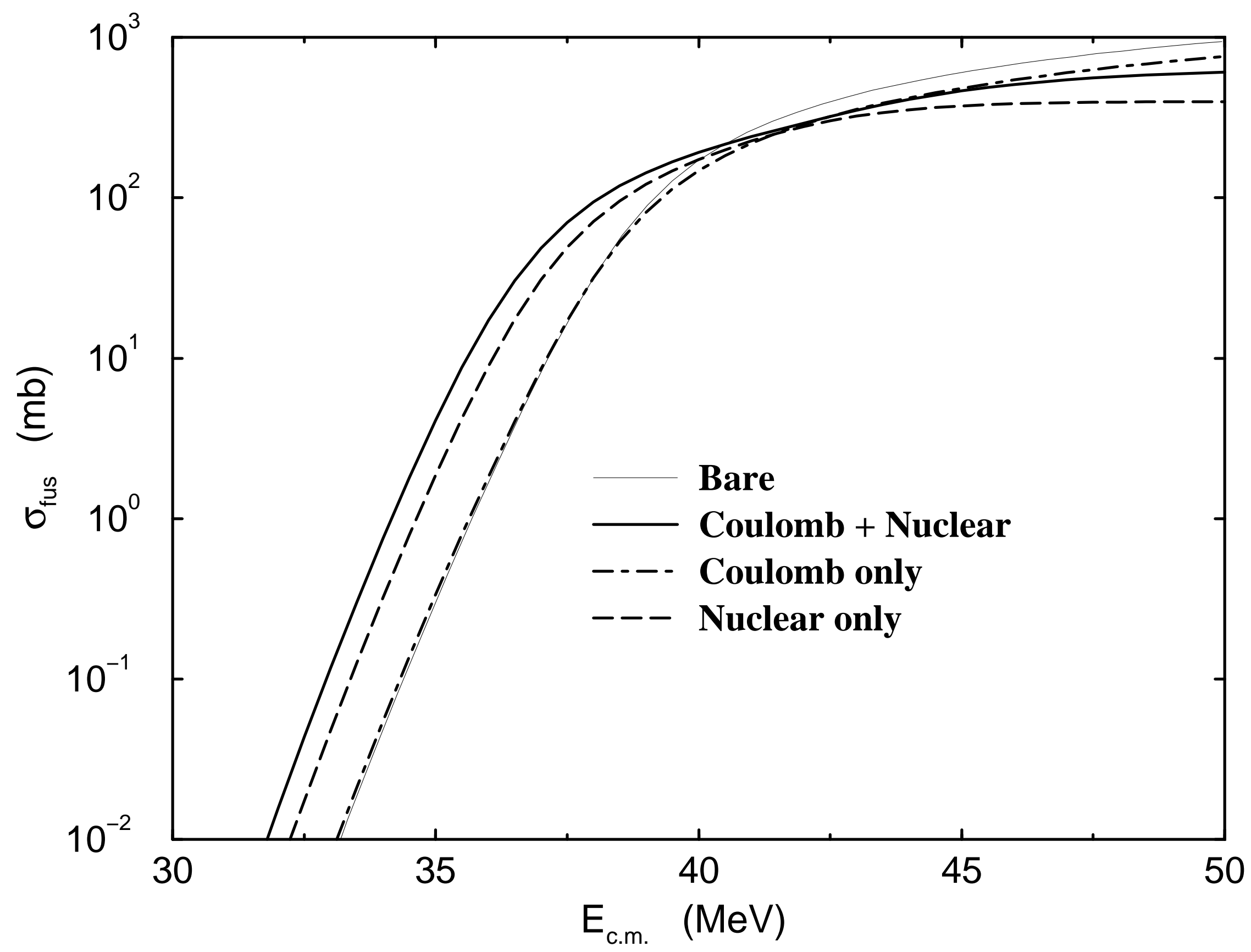

